\section{Escenario clínico}

Un paciente asmático de 20 años es atendido por su médico de familia. Está medicado con corticoides inhalatorios y agonistas beta 2 con buen control. Sin embargo, le comenta a su médico que tiene familiares que han realizado tratamientos con "vacunas" con el alergista y que, a partir de esto, no han requerido más medicación.

\section{Pregunta que generó el caso}

En pacientes asmáticos (población), ¿la inmunoterapia (intervención), mejora la función pulmonar y/o disminuye el requerimiento de medicación inhalatoria (resultados)?

\section{Estrategia de búsqueda}

Se realizó una búsqueda a través de Bireme (www.bireme.br) en la Biblioteca Cochrane empleando como palabras clave Asthma AND allergen AND therapy.De los 34 documentos hallados como revisión sistemática, hubo uno que parecía adecuado para responder la pregunta.

\section{El problema}

La inmunoterapia alergeno específica ("desensibilización") ha despertado controversias con relación a su utilidad en el manejo del asma bronquial.Algunos ensayos controlados y aleatorizados han demostrado efectos beneficiosos en resultados clínicamente importantes, pero con riesgo de reacciones anafilácticas serias e incluso fatales.

La inmunoterapia involucra la inyección de un extracto del alergeno bajo la piel con el objetivo de producir desensibilización.

Un metanálisis de 20 estudios (anterior al que se comenta en este EOP) encontró que los pacientes asignados a inmunoterapia tenían 3,2 veces más probabilidad de reportar menos síntomas de asma, 4,2 veces más probabilidad de requerir menos medicamentos para el asma y 6,8 veces más probabilidad de presentar disminución de la hiperreactividad bronquial que aquellos asignados a placebo.

Durante los últimos años ha habido interés creciente en nuevas preparaciones de alergenos y nuevas vías de administración.

Diferentes Guía de Práctica clínica para el manejo del asma reservan un papel secundario para la inmunoterapia argumentando que se desconocen sus efectos a largo plazo, el riesgo de anafilaxia y sus ventajas en comparación con el tratamiento estándar.

Resumen de la evidencia: Abramson MJ, Puy RM, Weinwr JM Inmunoterapia con alergenos para el asma (Cochrane Review). In:The Cochrane Library, Issue 3, 2003. Oxford: Update Software.

Objetivo: evaluar los efectos de la inmunoterapia alergeno específica en el tratamiento del asma.

Estrategia de búsqueda:se realizó una búsqueda en el registro Cochrane de Ensayos del Grupo de Vía Respiratorias (Cochrane Airways Group Trials Register) hasta 1997, en resúmenes de tesis, en WorldCat y ArticleFirst.

También se realizó una búsqueda en MEDLINE desde 1966 hasta 1997. Se buscaron las citas que contenían los artículos hallados a través de MEDLINE.Se buscaron estudios recientes publicados o no en WorldCat, ArticleFirst y resúmenes de disertación.

Métodos de revisión: la inclusión de los estudios se decidió por mayoría simple de tres revisores independientes. Las comparaciones planeadas fueron:

Inmunoterapia con alergenos vs. Placebo.

Inmunoterapia con alergenos vs. control antigénicamente inactivado.

Inmunoterapia con alergenos vs.control no tratado.

Polvo doméstico vs. placebo.

Se incluyeron ensayos con asignación al azar.

Resultados principales:se incluyeron cincuenta y cuatro ensayos. 25 trabajos eran de inmunoterapia para alergia contra ácaros, 13 ensayos de alergia al polen, ocho de alergia a epitelio de animales, dos de alergia al moho Cladosporium y seis sobre múltiples alergenos. El enmascaramiento en la asignación a cada rama de tratamiento se evaluó claramente en 11 trabajos. En varias comparaciones se encontró heterogeneidad significativa.

Síntomas: 16 estudios reportaron puntuaciones para síntomas. La diferencia de medias estandarizadas* (DME) fue de -0,52 (IC 95\% $-0,70$ a $-0,35)$ indicando una reducción estadísticamente significativa de los síntomas de asma en el grupo inmunoterapia. Sin embargo hubo heterogeneidad en los resultados.

21 estudios reportaron los síntomas como peores, iguales o mejores. El OR combinado fue de 0,27 (IC 95\% 0,21 a 0,35) a favor del grupo inmunoterapia. También hubo heterogeneidad para este resultado.

Uso de medicación: las puntuaciones de medicación para el asma fueron reportadas en siete estudios. La DME fue -0,51 (IC 95\% $-0,74$ a $-0,28$ ) indicando una reducción en el empleo de medicación antiasmática en la rama inmunoterapia. Los requerimientos de medicación se reportaron como aumentados, sin cambios o disminuidos en 15 estudios. El OR fue de 0,28 (IC 95\% 0,19 a 0,42) es decir que los pacientes aleatorizados a inmunoterapia requerían menos medicación. Hubo heterogeneidad significativa entre los siete estudios que reportaron puntuaciones de medicación.

Función pulmonar: se reportó este resultado en diez estudios. Ninguno incluyó el desvío estándar del VEF1. No hubo diferencias entre los grupos de tratamiento. Hubo heterogeneidad significativa entre los estudios.

Conclusión de los revisores: la inmunoterapia puede reducir los síntomas del asma y el empleo de medicación antiasmática. La magnitud del beneficio comparada con otros tratamientos es desconocida. Debe considerarse la posibilidad de eventos adversos serios como la anafilaxia.

\section{Comentario}

El presente metaanálisis ha encontrado que la inmunoterapia para el asma puede reducir los síntomas y disminuir los requerimientos de medicación.La heterogeneidad de las puntuaciones de síntomas y medicación puede deberse a los diversos esquemas de puntuación que se emplearon en los diferentes ensayos dado que cuando los resultados se reportaron en categorías hubo homogeneidad en los resultados. Es recomendable estandarizar las pun- 
tuaciones de síntomas y requerimiento de medicación en los estudios a futuro a fin de facilitar su interpretación. Cuando se evaluó la necesidad de medicación, sucedió algo parecido:al analizar categorías (requerimiento de medicación igual, menor o mayor) no hubo heterogeneidad y se concluyó que la inmunoterapia disminuyó el requerimiento de medicamentos. Este resultado podría ser de utilidad en la práctica si consideramos que la disminución de dosis de medicación es uno de los objetivos de la inmunoterapia con alergenos.Si bien el tratamiento con corticoides es el pilar fundamental en el tratamiento del asma bronquial, si una intervención lograra disminuir el requerimiento de esteroides manteniendo la función pulmonar a un costo y riesgos razonables; sería muy bienvenida.

Los resultados provenientes de un número pequeño de estudios con controles diferentes al placebo, deberían tomarse con cautela. Los estudios que emplean una rama "no tratamiento" son especialmente susceptibles a presentar sesgos y sobreestimar la magnitud del resultado. Los resultados sobre síntomas, medicación y función pulmonar encontrados en uno de estos estudios están por encima del límite superior del intervalo de confianza de los estudios controlados con placebo.

Dado el sesgo existente a favor de la publicación de estudios que encuentran diferencias entre las ramas (estudios positivos) es probable que existan estudios negativos que no se hayan publicado. Una manera de resolver este problema a futuro es que se registren todos los ensayos de inmunoterapia con alergenos. Lamentablemente, la calidad metodológica de los estudios no se pudo determinar claramente a través de la información contenida en los trabajos.

\section{Recomendación}

Si bien este metanálisis sugiere que la inmunoterapia podría reducir los síntomas y la demanda de drogas en pacientes asmáticos, si se lo compara con las terapias claramente beneficiosas, la magnitud de este beneficio no está todavía establecida. No puede saberse si el beneficio es igual en los pacientes tratados con esteroides que en los no tratados.

Tal vez el empleo más apropiado de la inmunoterapia sea en el asma claramente extrínseca y cuando el alergeno sea inevitable. No debe soslayarse el hecho de que la inmunoterapia puede acarrear efectos adversos serios e incluso fatales.

Existen numerosos interrogantes que no están suficientemente aclarados y que sería deseable que sean abordados por futuras líneas de investigación: ¿qué tipo de pacientes responde mejor? ¿es mejor la inmunoterapia monocomponente que el empleo de una combinación de alergenos? ¿cuál es la duración óptima del tratamiento? ¿cuál es el perfil de beneficio para los riesgos?.

Pero sin duda, la pregunta más relevante es encontrar el verdadero beneficio de la inmunoterapia, comparada con el tratamiento estándar con corticoides inhalatorios (que ha demostrado disminución de la morbimortalidad por asma) definiendo también cuál sería el rol de la inmunoterapia en pacientes que ya reciben corticoides. Con relación a la pregunta que nos generó el caso, la respuesta no es del todo contundente.Nuestro paciente podría requerir menos medicación (no sabemos cuánto menos y si ese "menos" es clínicamente relevante) si realizara inmunoterapia. Tampoco queda claro si la magnitud de este beneficio justifica los costos y potenciales efectos adversos.

Dra. Karin Kopitowski [ Unidad de Medicina Familiar y Preventiva. Hospital Italiano de Buenos Aires ]
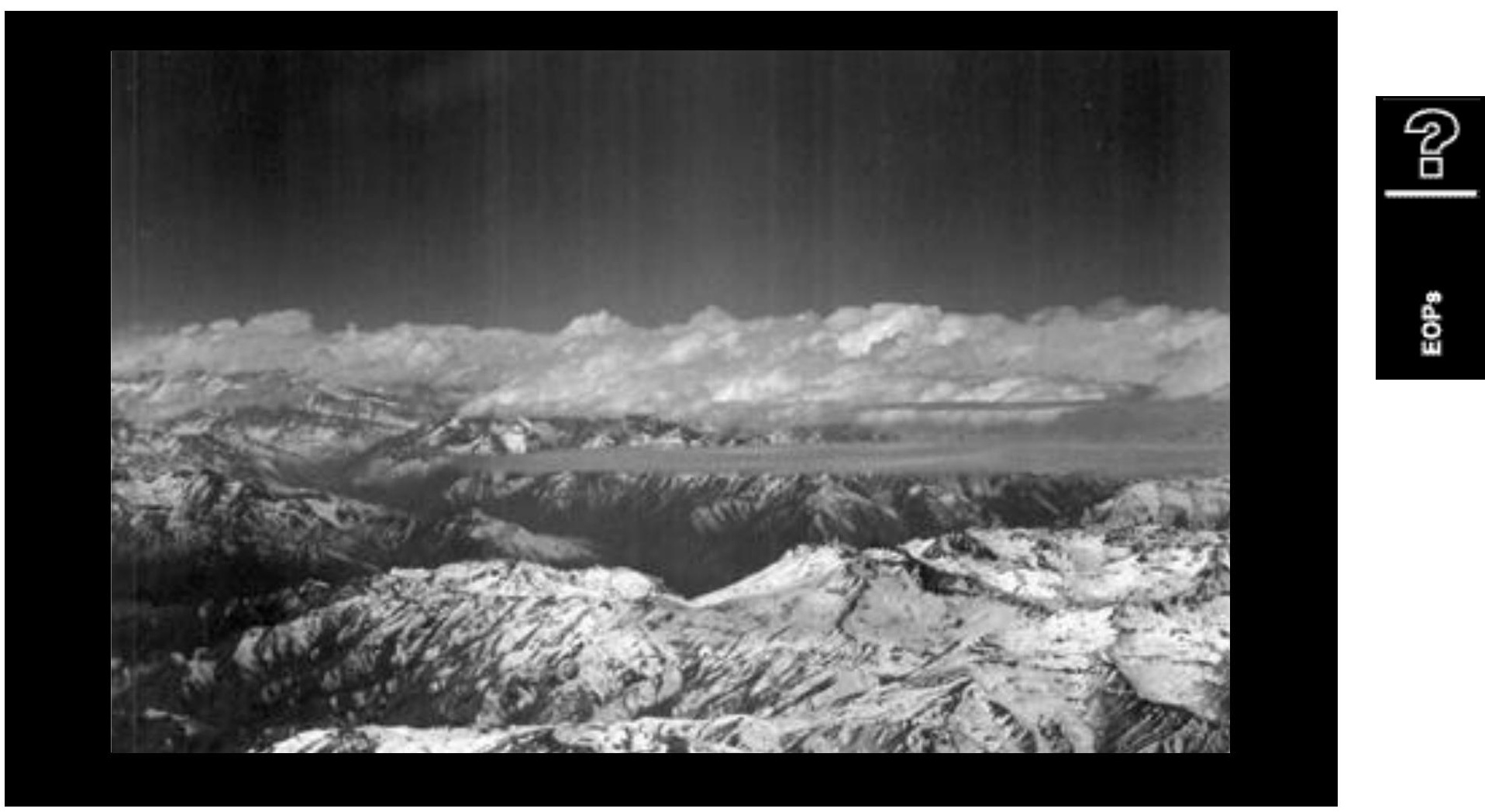